\title{
Sedation and Anesthesia for Magnetic Resonance Imaging during the COVID-19 Pandemic: Perspective
}

\author{
Kamath Sriganesh ${ }^{1}$ \\ ${ }^{1}$ Department of Neuroanaesthesia and Neurocritical Care, National \\ Institute of Mental Health and Neurosciences, Bengaluru, \\ Karnataka, India
}

J Neuroanaesthesiol Crit Care:2020;7:163-165

\section{Introduction}

Coronavirus disease 2019 (COVID-19) is a global pandemic affecting both community and healthcare system. Most children, adults with claustrophobia, neurological and psychiatric issues, and who are uncooperative or unable to lie still, require sedation or general anesthesia (GA) for facilitating magnetic resonance imaging (MRI) study. There is currently no guidance for sedation/anesthesia management for MRI during COVID-19 pandemic. This article discusses issues and approaches to periprocedural care of patients during MRI.

\section{Key Issues during Sedation or Anesthesia for MRI}

During the COVID-19 pandemic, it is difficult to differentiate non-COVID-19 from suspected/confirmed COVID-19 patient, in the absence of influenza-like illness (ILI), severe acute respiratory infection (SARI), or positive COVID-19 test. In India, guidelines for COVID-19 testing are provided by Ministry of Health and Family Welfare (MOHFW). ${ }^{1}$ Since the criteria are clear but rigid, no asymptomatic COVID-19 patients are eligible for COVID-19 testing unless they are primary contacts of confirmed COVID-19 patient (this is relaxed recently). Hence, clinical suspicion is warranted to prevent potential transmission of COVID-19 to healthcare providers (HCPs) and other patients.

Due to supply constraints, rational use of personal protective equipment (PPE) is recommended in non-COVID-19 hospitals and non-COVID-19 areas of hospitals with COVID-19 block. ${ }^{2}$ The MOHFW guidelines designate imaging services as areas at mild risk of COVID-19 transmission. Hence, only triple-layer mask and latex gloves are recommended. Many patients with neurological illness need sedation/GA for MRI procedure, some of whom may require elective/emergency airway management, which is an aerosol-generating procedure (AGP). All AGPs in suspected/confirmed COVID-19 patients require higher level of PPE that includes goggles/face shields,
Address for correspondence Kamath Sriganesh, DM, Department of Neuroanaesthesia and Neurocritical Care, National Institute of Mental Health and Neurosciences, Bengaluru 560029, Karnataka, India (e-mail: drsri23@gmail.com).

N95 mask, and gloves. Additionally, coveralls and shoes covers are desirable as splashes of airway secretions and vomitus are likely during airway management.

All components of the PPE (goggle, face-shield, N95 mask with metal clip or respirator) should be checked for presence of ferromagnetic materials and replaced where feasible with tape before donning, as these may be MRI noncompatible or untested. ${ }^{3}$ Powered air-purifying respirator is unsuitable for zone 4 of MR environment. ${ }^{4}$ Increasing air change rates per hour (ACH) especially during AGPs becomes important as high-efficiency particulate air filtration system may not be suitable for MR environment. Higher $\mathrm{ACH}$ also ensures minimum downtime after imaging of a suspect/positive COVID-19 patient.

\section{Suggested Approach for Testing and Personal Protection}

MRI study is mostly an elective procedure and may be postponed when possible. ${ }^{5}$ All stakeholders should understand implications of conducting MRI during the pandemic. Need for sedation/anesthesia should also be judiciously considered.

Both COVID-19 and non-COVID-19 patients may require MRI study during this pandemic. A dedicated MRI facility for suspected/confirmed COVID-19 patients may be appropriate wherever feasible (red-zone). This minimizes contact between COVID-19 infected and noninfected patients/HCPs, minimizes time for infection control measures between patient care, and ensures efficient utilization of human and material resources. If this is not feasible, separate pathway should be created for movement of COVID-19 patients. Thorough disinfection of pathway and MRI facility with recommended disinfectants should be performed after imaging.

If MRI under sedation/GA is deemed necessary (but not emergency), patients with ILI/SARI should be first tested for COVID-19 and MRI should be performed after negative report and after clinical symptoms subside.
DOI https://doi.org/ $10.1055 / \mathrm{s}-0040-1718972$ ISSN 2348-0548. (c) 2020. Indian Society of Neuroanaesthesiology and Critical Care. This is an open access article published by Thieme under the terms of the Creative Commons Attribution-NonDerivative-NonCommercial-License, permitting copying and reproduction so long as the original work is given appropriate credit. Contents may not be used for commercial purposes, or adapted, remixed, transformed or built upon. (https://creativecommons.org/licenses/by-nc-nd/4.0/).

Thieme Medical and Scientific Publishers Pvt. Ltd., A-12, 2nd Floor, Sector 2, Noida-201301 UP, India 
If MRI is deemed emergent and the patient is suspected/confirmed of COVID-19, risk for transmission of COVID-19 infection should be considered. Full PPE should be worn by all HCPs for the entire duration starting from preanesthetic evaluation and airway assessment, obtaining of consent, placement of intravenous access, attachment of monitors, administration of sedation/anesthesia, conduct of MRI and observation in postanesthesia care unit till the discharge from MRI facility.

If the report is negative (especially rapid antigen) but patient displays ILI/SARI symptoms, appropriate PPE (goggles/face shields, N95 mask, and gloves) should be considered as even reverse-transcription polymerase chain reaction test may have sensitivity of only $70 \%{ }^{6}$

If COVID-19 is not suspected, routine precautions during COVID-19 times such as hand washing with soap and water or alcohol-based disinfectant before and after patient care, maintaining minimum physical contact with patient, and wearing of mask may be sufficient.

\section{Suggested Sedation/Anesthesia Technique for MRI}

The technique of sedation/ anesthesia in suspected/confirmed COVID-19 patients may require modifications during this pandemic.

GA with intubation predisposes to aerosol generation at multiple time points-mask ventilation, intubation, ventilation, extubation, and suctioning of secretions. ${ }^{7}$ Intubation is rarely needed for MRI even during non-COVID-19 times. Therefore, when possible, it should be avoided. Intubation if performed should be preferably with disposable laryngoscope blades. If patient arrives intubated, cuff pressure should be checked and maintained between 25 and $30 \mathrm{cmH} 2 \mathrm{O}$ to prevent leak. Disposable breathing circuit and two viral filters, at patient-end and machine-end, should be used to minimize contamination of anesthesia workstation. A closed suction catheter system should be used to remove airway secretions.

GA with supra-glottic airway devices (SGADs) may also require reconsideration. SGADs do not always provide good seal, result in leak of aerosols, and predispose to increased oropharyngeal secretions from airway handling. When used, SGAD should be placed and removed under sedation to avoid coughing, unless contraindicated. Viral filters, disposable circuits, and spontaneous respiration with either inhalational (sevoflurane) or intravenous (propofol) anesthesia should be considered.

The most commonly practiced technique for MRI is intravenous sedation with dexmedetomidine or propofol infusion avoiding airway instrumentation. ${ }^{8}$ The same may be considered during COVID-19 pandemic. Bradycardia and hypotension are likely with dexmedetomidine; hence, their presence is a contraindication for its use. Propofol, mostly after bolus doses, can result in airway collapse, apnea, desaturation, and hypotension. It is contraindicated in patients with known hypersensitivity. Challenges of securing an emergency airway in the MRI facility in suspected/confirmed COVID-19 patients should be understood. Planning and preparation for such eventuality should be in place including an isolated intubation area preferably with negative pressure.

\section{Other Considerations}

The possibility of patient contracting COVID-19 infection from HCPs and vice-versa during care in MRI facility exists. This should be explained as a disclaimer to the patient during consent. In nonintubated patients, triple-layer mask must be placed to cover nose and mouth. Oxygen mask can be placed over the triple-layer mask while nasal prongs should be paced below. Low-flow oxygen should be used to maintain oxygen saturation $>94 \%$. Sampling tube for monitoring respiration and carbon dioxide levels may be placed between patient's mask and oxygen mask. A viral filter should be placed at the monitor end, and sampling tube and oxygen mask should be disposed after the procedure. Electrocardiogram cable and blood pressure cuff should be cleaned with surface disinfectant ( $\geq 70 \%$ isopropyl alcohol) and reused. Frequent contact with the patient during MRI study should be avoided by administering sedation as an infusion (with MRI-compatible pump) rather than boluses. Deep sedation can cause airway obstruction, necessitates repeat imaging and airway maneuvers; hence, should be avoided. In contrast, lighter sedation leads to patient movement. Therefore, appropriate titration of sedation/anesthetic drug is required. A slave monitor helps continuous remote monitoring of the patient during MRI and ensures safety of both patients and HCPs. Minimum number of HCPs with appropriate PPEs should come in contact with patient during MRI. Communication and visibility may be difficult with full PPE and hence reconfirmation is necessary to avoid errors. Lastly, HCPs should be rotated between COVID-19 and non-COVID-19 areas to prevent stress and anxiety, and to maintain their well-being.

\section{Conclusion}

Providing sedation/anesthesia for MRI procedure during COVID-19 pandemic is challenging. Good planning and preparation and effective implementation of strategies appropriate for COVID-19 times are necessary to achieve good outcomes for patients and HCPs in this unprecedented time.

\section{Conflict of Interest}

None declared.

\section{References}

1 Strategy for COVID-19 testing in India. Available at: https:// www.mohfw.gov.in/pdf/Revisedtestingguidelines.pdf. Accessed on June 18, 2020

2 Novel Coronavirus Disease 2019 (COVID-19): Additional guidelines on rational use of Personal Protective Equipment (setting approach for Health functionaries working in non-COVID areas). Available at: https://www.mohfw. gov.in/pdf/AdditionalguidelinesonrationaluseofPersonal ProtectiveEquipmentsettingapproachforHealthfunctionaries workinginnonCOVIDareas.pdf. Accessed June 18, 2020 
3 Drum E, McClung Pasqualino H, Subramanyam R. Anesthesia and potential aerosol generation during magnetic resonance imaging in children with COVID-19. Paediatr Anaesth 2020;30(8):944-946

4 ACR guidance on COVID-19 and MR use. Available at: https://www.acr.org/Clinical-Resources/Radiology-Safety/ MR-Safety/COVID-19-and-MR-Use? fbclid=IwAR3AwjCgeEkIWKy3UbbRkq_sl9dV_CLRPoPXjIA4OtM25nwzBsX2FilywY. Accessed September 21, 2020

5 Jangra K, Manohar N, Bidkar PU, et al. Indian Society of Neuroanaesthesiology and Critical Care (ISNACC) Position Statement and Advisory for the Practice of Neuroanesthesia during COVID-19 Pandemic Endorsed by Indian Society of
Anaesthesiologists (ISA). J Neuroanaesthesiol Crit Care 2020. Doi: $10.1055 / \mathrm{s}-0040-1714186$

6 Arevalo-Rodriguez I, Buitrago-Garcia D, SimancasRacines D, et al False-negative results of initial RT-PCR assays for covid-19: a systematic review. Medrxiv 2020. Doi: $10.1101 / 2020.04 .16 .20066787$

7 Sriganesh K, Rao GSU. COVID-19: the unseen threat for the healthcare professionals. J Neurosci Rural Pract 2020; 11(3):367-368

8 SriganeshK, SainiJ, Theerth K,Venkataramaiah S.Airway dimensions in children with neurological disabilities during dexmedetomidine and propofol sedation for magnetic resonance imaging study. TurkJAnaesthesiol Reanim 2018;46(3):214-221 